\title{
Galileo Single Frequency Ionospheric Correction: \\ Performances in Terms of Position
}

\author{
Benoît Bidaine, Matthieu Lonchay \\ Fund for Scientific Research - FNRS \\ University of Liège (ULg) - Geomatics Unit, Allée du 6 Août 17, 4000 Liège, \\ Belgium \\ B.Bidaine@alumni.ulg.ac.be \\ http://www.geo.ulg.ac.be/ \\ René Warnant \\ University of Liège (ULg) - Geomatics Unit, Allée du 6 Août 17, 4000 Liège, \\ Belgium
}

Keywords Galileo ; positioning ; ionospheric correction ; single frequency ; NeQuick model ; data ingestion

\begin{abstract}
For GPS single frequency users, the ionospheric contribution to the error budget is estimated by the well-known Klobuchar algorithm. For Galileo, it will be mitigated by a global algorithm based on the NeQuick model. This algorithm relies on the adaptation of the model to slant Total Electron Content (sTEC) measurements. Although the performance specifications of these algorithms are expressed in terms of delay and TEC, the users might be more interested in their impact on positioning. Therefore, we assessed the ability of the algorithms to improve the positioning accuracy using globally distributed permanent stations for the year 2002 marked by a high level of solar activity. We present uncorrected and corrected performances, interpret these and identify potential causes for Galileo correction discrepancies. We show vertical errors dropping by 56-64 \% due to the analyzed ionospheric corrections, but horizontal errors decreasing by $27 \%$ at most. By means of a fictitious symmetric satellite distribution, we highlight the role of TEC gradients in residual errors. We describe mechanisms permitted by the Galileo correction, which combine sTEC adaptation and topside mismodeling, and limit the horizontal accuracy. Hence, we support further investigation of potential alternative ionospheric corrections. We also provide an interesting insight into the ionospheric effects possibly experienced during the next solar maximum coinciding with Galileo Initial Operation Capability.
\end{abstract}

Bidaine and Warnant - doi:10.1007/s10291-012-0261-0 


\section{Introduction}

Since Global Navigation Satellite Systems (GNSS) signals travel through the atmosphere, GNSS receiver operation largely depends on the dynamics of the ionosphere. Most civilian receivers model the ionospheric delay to mitigate its effect on positioning. When processing single frequency measurements only, they cannot compensate for ionospheric errors by taking advantage of ionosphere dispersion causing differential effects between frequencies. They run an internal model fed with external information about the state of the ionosphere disseminated by the navigation system. Hence, the Global Positioning System (GPS) broadcasts 8 coefficients suited for the ionospheric correction algorithm (ICA) designed by Klobuchar (1987). The future Galileo system will transmit 3 coefficients $a_{0}, a_{1}$ and $a_{2}$ dedicated to the NeQuick model, the core of the socalled Galileo single frequency ionospheric correction algorithm (HofmannWellenhof et al. 2008). For the solar activity input of the model, these coefficients define an "effective ionization level" $A z$ as a second-order polynomial of the receiver modified dip-latitude (modip) $\mu$,

$$
A z=a_{0}+a_{1} \mu+a_{2} \mu^{2}
$$

with

$$
\tan \mu=\frac{I}{\sqrt{\cos \varphi}}
$$

being a function of the geomagnetic inclination $I$ and the geographic latitude $\varphi$ (Rawer 1963). The coefficients will be issued daily by the Galileo Mission Segment based on data adaptation via an optimization procedure to find the best input for the model to fit a specific dataset. This procedure will be performed at each Galileo Sensor Station (GSS): Each GSS will routinely measure the slant Total Electron Content (slant TEC or sTEC) toward each satellite and compute the $A z$ value minimizing the root mean square (RMS) difference between measured and modeled sTEC,

$$
R M S=\sqrt{\left\langle\left(s T E C_{\text {mod }}(A z)-s T E C_{\text {meas }}\right)^{2}\right\rangle}
$$


Future GNSS single frequency applications will depend on the capability of the GPS and Galileo algorithms to account for the ionospheric delay but even more to reduce positioning errors. Consequently we investigated the positioning accuracy reached with ionospheric corrections supplied by these algorithms. For Galileo, we considered three different versions of the underlying NeQuick model: NeQuick 1 (Radicella and Leitinger 2001), which is publicly available from the ITU-R website (ITU-R Rec. P.531; http://www.itu.int/oth/R0A04000018/en), NeQuick G, which will be implemented in Galileo and was kindly provided by the European Space Agency (ESA), and NeQuick 2 (Nava et al. 2008), furnished by the model designers at the Abdus Salam International Centre for Theoretical Physics (ICTP). We performed stand-alone point positioning from GPS single frequency code measurements. We used the actual broadcast coefficients from GPS RINEX files to apply the Klobuchar algorithm. Regarding NeQuick, we simulated them for the In-Orbit Validation (IOV) phase of Galileo (Bidaine and Warnant 2011). We selected 18 stations belonging mainly to the International GNSS Service (IGS) (Dow et al. 2009) to represent the GSS (Fig. 1). At these stations, we adapted the model to sTEC values calibrated by means of IGS Global Ionospheric Maps (GIM) (Orus et al. 2007b; Hernández-Pajares et al. 2009).

We discuss the impact of several real and simulated single frequency ionospheric corrections on positioning. First we characterize the correction performances for the high solar activity year 2002. Second we propose an intuitive interpretation of these performances in particular for horizontal positioning. Third we identify different potential origins for the observed discrepancies of the Galileo correction.

\section{Ionospheric correction performances}

We derived ionospheric correction performances studying 8 test sites. These sites cover all modip bands (Fig. 1) and should therefore involve ionospheric conditions characteristic for low, mid- and high latitudes at solar maximum. The associated GPS stations (Table 1) belong to the IGS, the European Reference Frame (EUREF) Permanent Network (Bruyninx 2004) or the Continuously Operating Reference Stations (CORS) network (Snay and Soler 2008) and provide 
at least $90 \%$ RINEX data availabilities for 2002. They also lie in the vicinity of ionosondes that are potentially useful for further investigation.

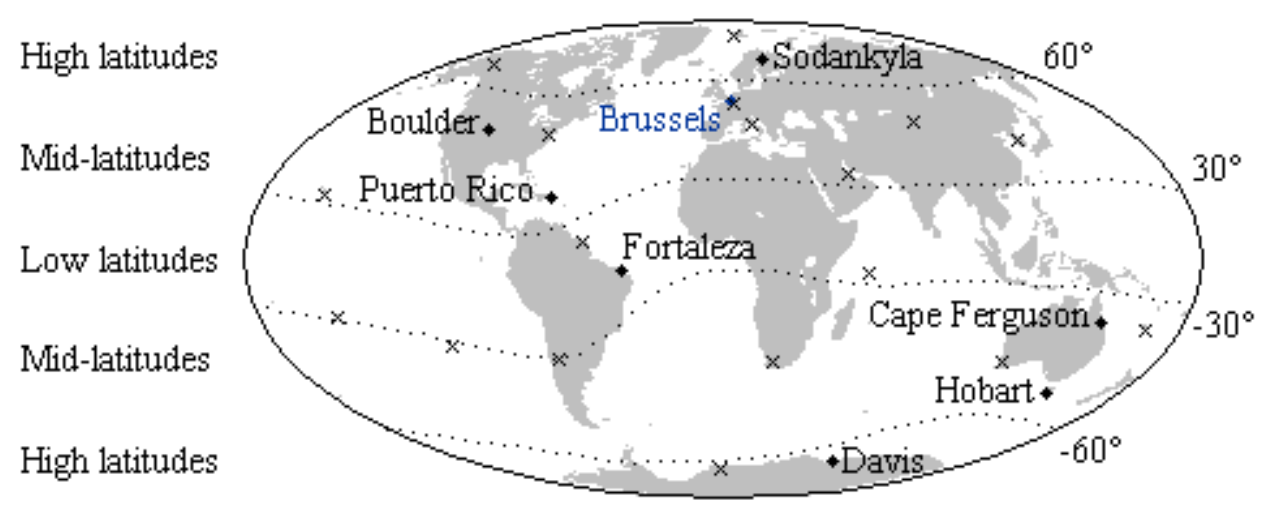

Fig. 1 Location of the GPS stations selected as test sites (dots) and representative IOV GSS (crosses). These stations cover all modip bands.

Table 1 Characteristics of the GPS stations selected as test sites.

\begin{tabular}{llll}
\hline Station & Code & Network & Modip $\left[^{\circ}\right]$ \\
\hline Sodankyla & SODA & EUREF & 65.2 \\
Brussels & BRUS & IGS/EUREF & 55.3 \\
Boulder & DSRC & CORS & 53.5 \\
Puerto Rico & PUR3 & CORS & 39.7 \\
& & & \\
Fortaleza & FORT & IGS/CORS & -8.7 \\
Cape Ferguson & TOW2 & IGS & -40.1 \\
Hobart & HOB2 & IGS & -55.3 \\
Davis & DAV1 & IGS & -64.3 \\
\hline
\end{tabular}

This section describes the performances of the Klobuchar and NeQuick models for the chosen sites and year. First we consider their effectiveness in terms of sTEC, which is the primary parameter they are intended to provide for each satellite in view. Moreover, this parameter-the equivalent ionospheric delay for the Klobuchar algorithm-is employed for dedicated system specifications. Then we focus on the positioning errors reduction enabled by the corrections which informs about services accuracy. Finally we address the spatio-temporal variability of the observed positioning performances. 
In order to analyze sTEC modeling, we compared modeled values to GPS-derived data calibrated by means of GIM. The latter reference sTEC measurements were generated from the geometry-free phase combination of which the ambiguities were computed using sTEC estimates from IGS maps (Orus et al. 2007b). From all the available differences between these reference measurements and corresponding modeled values for the selected year and sites, we calculated the relative RMS errors (Fig. 2): We divided the absolute RMS errors by the mean measured sTEC which amounts to 44 TECU (1 TECU equals $10^{16} \mathrm{~m}^{-2}$ and induces an error of $0.16 \mathrm{~m}$ on the $\mathrm{L} 1$ carrier).

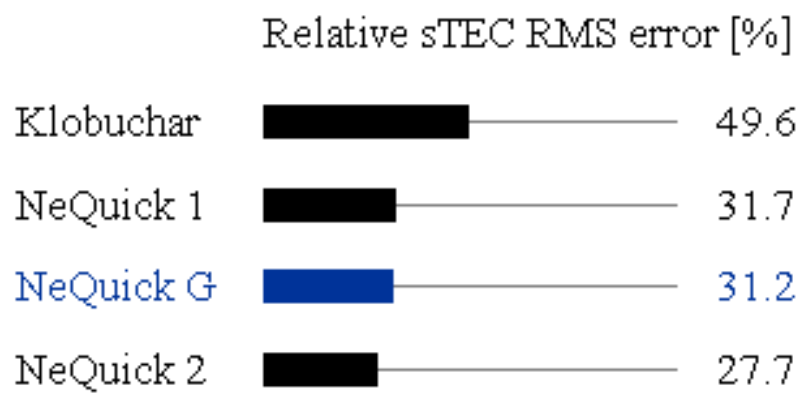

Fig. 2 Relative sTEC RMS error obtained with the ionospheric corrections in 2002. The NeQuick model exhibits better performances in terms of sTEC by comparison with the Klobuchar model.

According to the sTEC statistics, NeQuick outperforms the Klobuchar model. Even if the Klobuchar model fits the declared performance of $50 \%$ RMS correction, it underestimates sTEC by 11 TECU and the standard deviation of its differences equals 19 TECU. NeQuick 1 and G yield biases 5 and 7 times smaller and $27 \%$ reduced standard deviations. In agreement with other studies (Orus et al. 2007a), NeQuick 2 significantly decreases the mean sTEC difference below 1 TECU and ends up with a 12-TECU standard deviation, that is, $11 \%$ less than the other versions.

NeQuick complies with the Galileo algorithm specifications for the stations and year of study. This algorithm has been designed to limit the residual error to $30 \%$ of the actual sTEC or 20 TECU, whichever is larger. In our simulation using NeQuick G, $93 \%$ of the residual errors meet this criterion (Fig. 3). This proportion is less than $1 \%$ smaller for NeQuick 1 and larger for NeQuick 2 and is 
consistent with previous results (Prieto-Cerdeira et al. 2006). By comparison with the other versions, the compliant data for NeQuick 2 include more large sTEC measurements-larger than $20 T E C u / 30 \% \approx 66.7 T E C u$, associated to large residual errors_-but less smaller sTEC measurements.

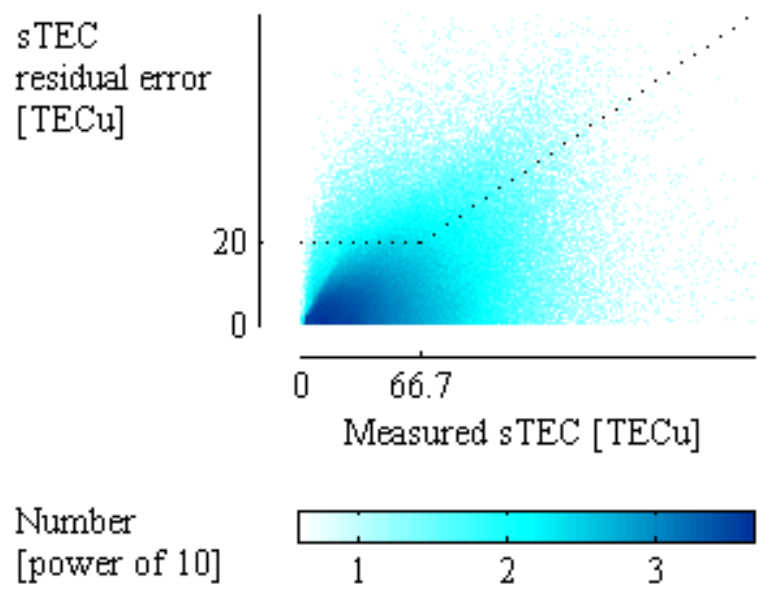

Fig. 3 sTEC residual errors from NeQuick G compared to the Galileo algorithm specifications in 2002. $93 \%$ of the residual errors meet the specifications (below the dotted line).

\section{Positioning errors}

The second performance analysis dealt with differences between the actual position of the test stations and the ones computed with and without each ionospheric correction. Thanks to the gLAB software tool suite (HernandezPajares et al. 2010), we performed single-point single-epoch (SPSE) positioning characteristic of the operation of a typical mass-market single frequency receiver: We used L1 C/A pseudorange measurements, broadcast orbits, clocks and hardware biases as well as a simple nominal tropospheric model and the Niell mapping function. As such this processing corresponds to a first "uncorrected" case. Applying additional pseudorange corrections accounting for the ionospheric delay using each model renders the four other cases. To this extent, we integrated adequate NeQuick-related instructions into gLAB. We synthesized the results in terms of horizontal and vertical $95 \%$ accuracies (Fig. 4), as these metrics are regularly used in services specifications, for example, 15 and $35 \mathrm{~m}$ for the Galileo single frequency open service (Hofmann-Wellenhof et al. 2008). 


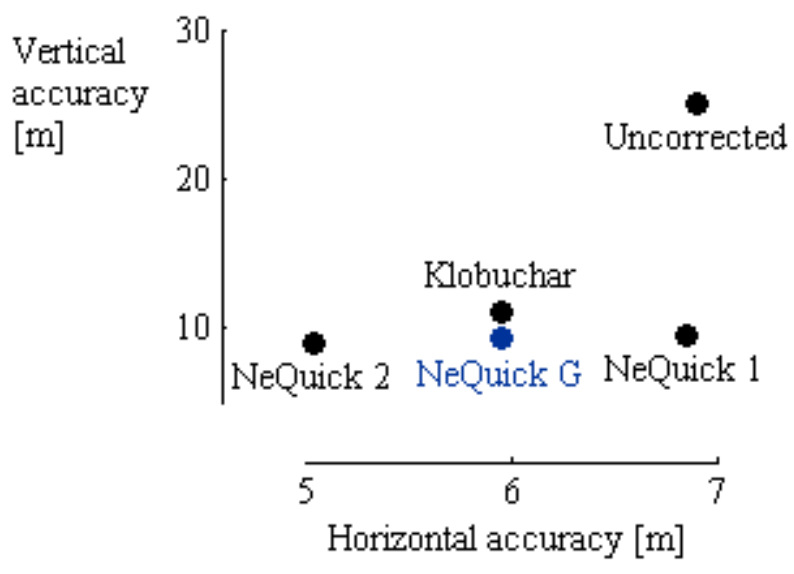

Fig. 4 Positioning accuracies obtained with and without the ionospheric corrections in 2002. The corrections improve more the vertical accuracy than the horizontal one. These numbers correspond to the 95th percentile of absolute horizontal and vertical positio

All the ionospheric corrections analyzed increase the positioning accuracy with respect to the uncorrected case at different levels, however, for the horizontal and vertical components. The vertical error $(25.1 \mathrm{~m})$ drops significantly for the Klobuchar algorithm (-56 \%) and even more with successive NeQuick versions ($62 \%,-63 \%$ and $-64 \%)$. On the other hand, the horizontal error $(6.9 \mathrm{~m})$ decreases much less with the Klobuchar, NeQuick G and 2 models $(-14 \%,-14 \%$ and $-27 \%$ ) but barely with NeQuick 1 . These results easily reach the target performance specifications of the Galileo single frequency open service. However they suggest to investigate the correlation between sTEC and positioning residual errors which is low (Fig. 5). They bring forward the influence of the satellite sky distribution explaining partially this decorrelation as well as the generally larger vertical errors by comparison to horizontal ones. Given the chosen year, these errors likely exceed those obtained for lower solar activity periods. Furthermore, they do not correspond to ionosphere mismodeling only. They are indeed largely affected by the accuracy of the various products implemented (orbits, clocks, hardware biases and troposphere) as well as code noise and multipath which should be smaller for Galileo than for the GPS data exploited for this simulation. 


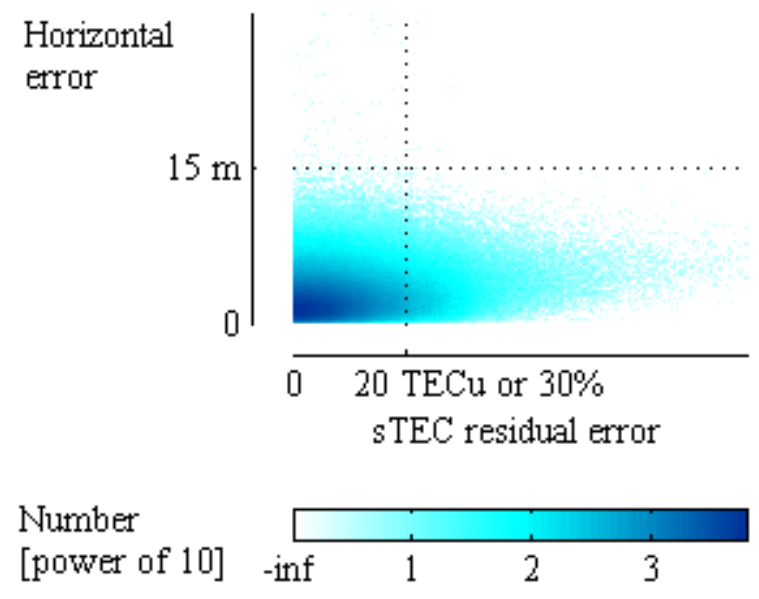

Fig. 5 Combined comparison of sTEC residual errors and horizontal residual errors from NeQuick $\mathrm{G}$ to the Galileo ionospheric correction algorithm and Open Service specifications in 2002. The sTEC specifications (on the left of the vertical dotted line) and the positioning requirements (below the horizontal dotted line) are met simultaneously for $93 \%$ of the residual errors. However, sTEC and horizontal errors outside specifications do not necessarily correspond to each other. The sTEC residual error axis consists in a hybrid scale using TECU for values associated with measured sTEC smaller than 66.7 TECU and \% otherwise. It enables a graphical comparison to the Galileo algorithm specifications (depicted by a straight line when aligning 20 TECU and $30 \%$ ) independently from the measured sTEC.

\section{Spatio-temporal variability}

Looking for explanations for the differences between the obtained positioning performances, we inspected their variations in space and time. In particular, we examined 95th-percentile dependencies on month, local time and modip, three variables conditioning the state of the ionosphere. As the effectiveness of the ionospheric corrections appears more questionable for horizontal positioning, we focused on this component.

The monthly horizontal accuracy shows a similar trend as the TEC, having maxima around the equinoxes (Fig. 6). It is uniformly improved by any of the ionospheric corrections considered apart by NeQuick 1, which exhibits larger values than the uncorrected case for about half of the year (November to March). It is the best with NeQuick 2 and better with NeQuick G than with Klobuchar for two-third of the year. 


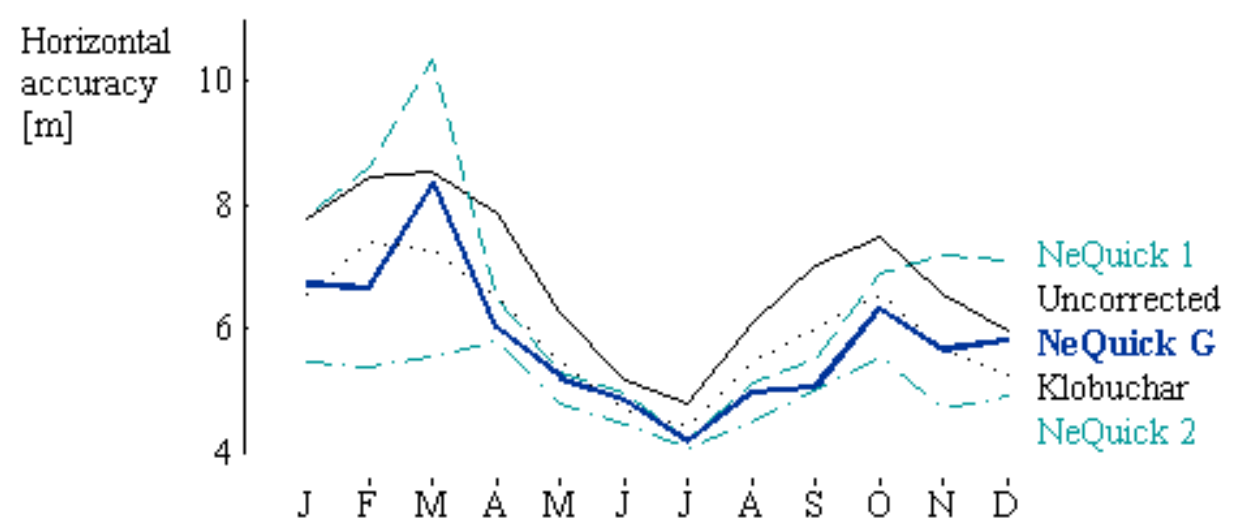

Fig. 6 Monthly variability of the horizontal accuracy obtained with and without the ionospheric corrections (continuous line for uncorrected case, dotted for Klobuchar, dashed for NeQuick 1, thick for NeQuick G and dash-dotted for NeQuick 2).

The intra-day variability of horizontal errors only partially resembles the TEC profile (Fig. 7). The uncorrected positioning errors rise faster than TEC in the morning and remain large in the evening. They are partially mitigated by all the corrections except by NeQuick 1 which amplifies them at night. They are handled at best by NeQuick 2. NeQuick G competes again with Klobuchar and especially involves discrepancies at nighttime.

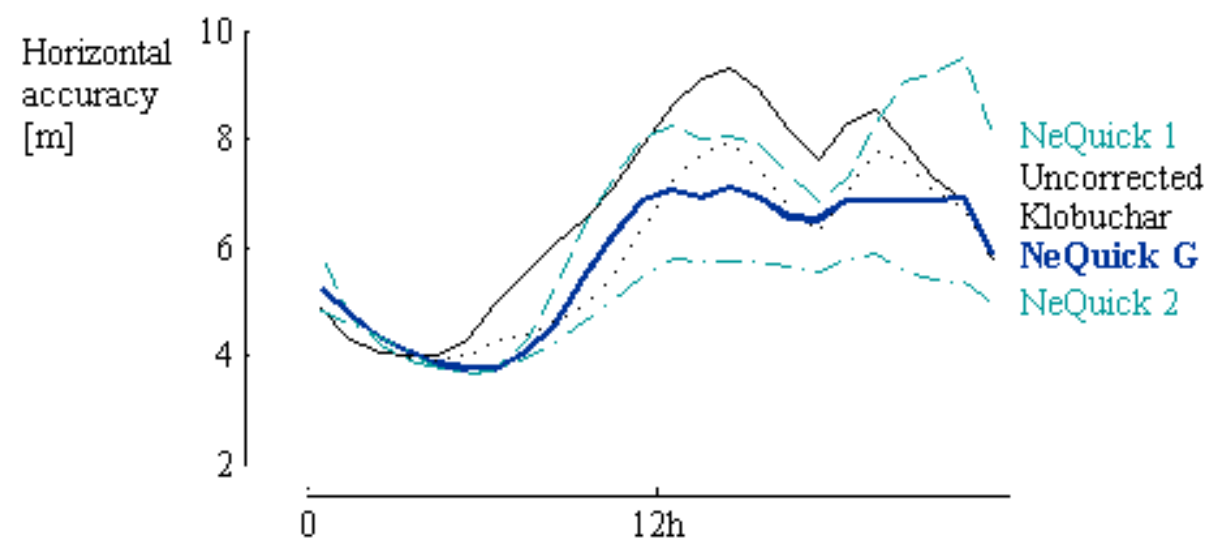

Fig. 7 Local-time variability of the horizontal accuracy obtained with and without the ionospheric corrections (continuous line for uncorrected case, dotted for Klobuchar, dashed for NeQuick 1, thick for NeQuick G and dash-dotted for NeQuick 2). 
The horizontal accuracy shows comparable orders of magnitude at different modip with or without correction with the largest range within the mid-latitude region. Its largest (resp. smallest) values appear at low (resp. high) mid-latitudes unlike TEC (Fig. 8). The models still provide a correction apart from NeQuick 1 which even adds errors at four stations including Fortaleza. Once more, NeQuick 2 is associated to the best accuracy in nearly all of the cases. NeQuick G and Klobuchar stand between the two other models. In particular, NeQuick G brings only little improvement at Brussels where NeQuick 1 worsens the horizontal accuracy. Hence, Brussels constitutes the worst of the selected sites in terms of positioning performances improvement from the Galileo correction with respect to the uncorrected case. Therefore, this station deserves the focus of the following sections interpreting the positioning performances and dealing with the Galileo correction discrepancies.

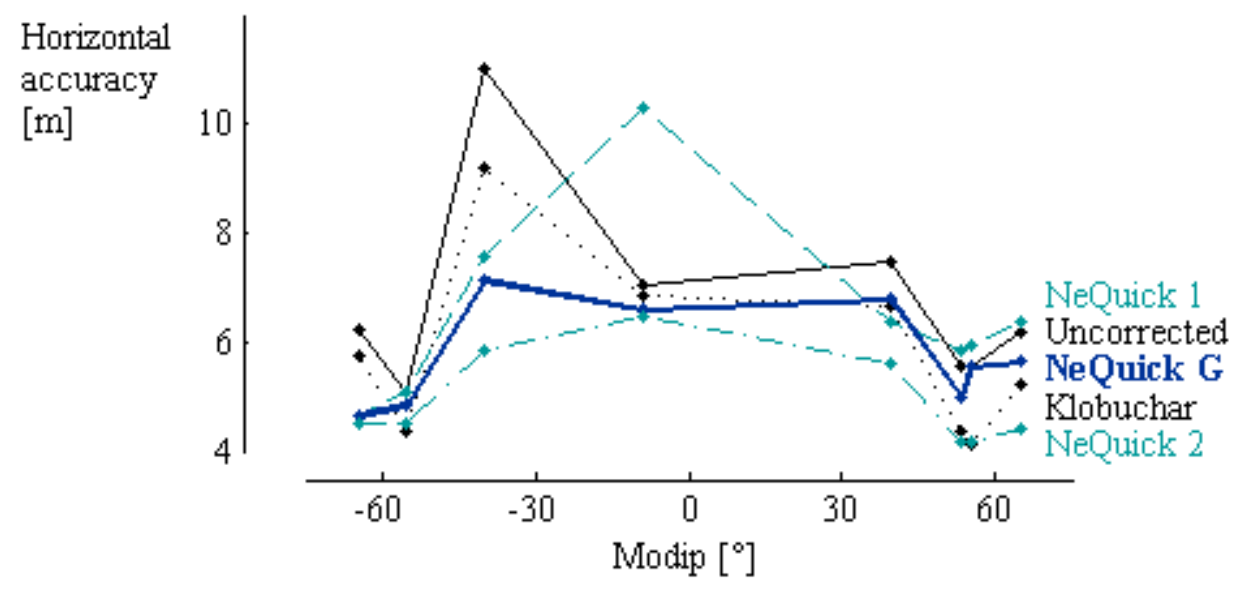

Fig. 8 Modip variability of the horizontal accuracy obtained with and without the ionospheric corrections (continuous line for uncorrected case, dotted for Klobuchar, dashed for NeQuick 1, thick for NeQuick G and dash-dotted for NeQuick 2).

\section{Interpretation}

Interpreting the impact of the ionosphere and its modeling on SPSE positioning is not straightforward. Positioning errors do not simply follow TEC trends (Fig. 8). They depend on the satellite sky distribution, each satellite-to-receiver link probing a different region of the ionosphere. Therefore, we assessed a single station (Brussels) below a smoother ionospheric region. We mainly covered horizontal errors for their rather small correction levels despite the good sTEC performances. 
This section addresses the way the ionospheric (residual) delay translates into final coordinates. It successively explains the influence of the ionosphere on uncorrected positions and discusses the conclusions applicability to corrected solutions.

\section{lonosphere influence on positioning}

The distributions of horizontal and vertical uncorrected positioning errors underlying the $95 \%$ accuracies described in the previous section reveal systematic trends at northern mid-latitudes (Fig. 9). They display average offsets toward the north and up directions. Yet negative heights would intuitively have ensued from ionospheric delays lengthening the pseudoranges from every satellite. The horizontal distribution is also characterized by a larger dispersion along the north axis than along the east one suggesting a larger role of the north error in horizontal accuracy.
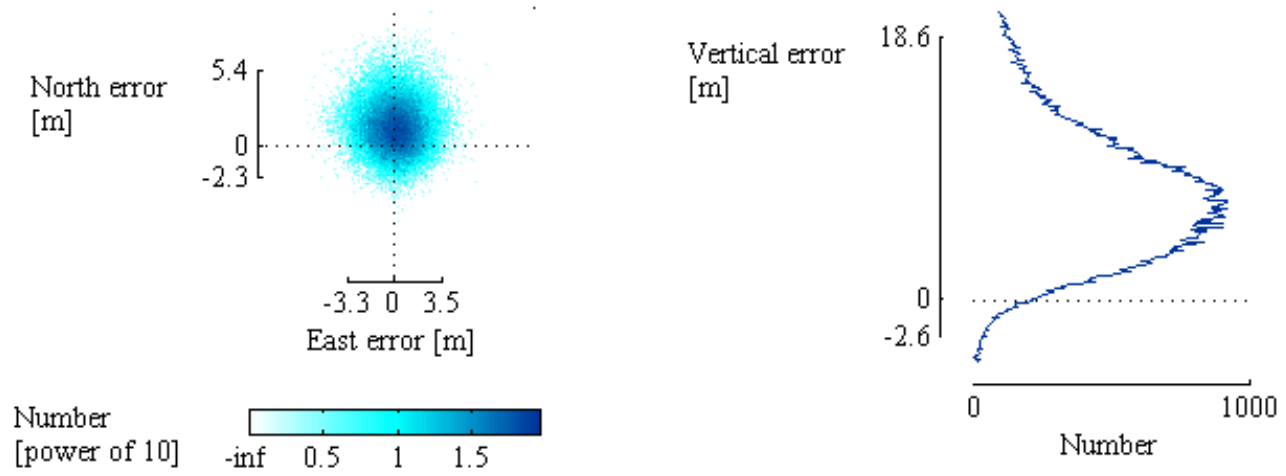

Fig. 9 Distributions of the horizontal (left plot) and vertical (right plot) uncorrected positioning errors at Brussels. The axes represent 2- $\sigma$ intervals around corresponding means.

We referred to the positioning mathematical problem to explain these tendencies (Hofmann-Wellenhof et al. 2008). This problem aims at determining the three coordinate differences $\Delta \mathbf{r}$ from a priori approximations and an unknown bias $\Delta b$ usually assimilated to the receiver clock error, all gathered in the vector $\Delta \mathbf{x}$. These unknowns relate to the differences $\Delta \mathbf{P}$ between the measured pseudoranges to the $\mathrm{n}$ satellites in view of the receiver and the corresponding values computed from the a priori coordinates as follows

$$
\Delta \mathbf{P}=\mathbf{A} \Delta \mathbf{x}+\mathbf{v}
$$


with

$$
\Delta \mathbf{P}=\left(\begin{array}{c}
\Delta P^{1} \\
\vdots \\
\Delta P^{n}
\end{array}\right), \mathbf{A}=\left(\begin{array}{cc}
-\mathbf{u}^{1} & 1 \\
\vdots & \vdots \\
-\mathbf{u}^{n} & 1
\end{array}\right), \Delta \mathbf{x}=\left(\begin{array}{c}
\Delta \mathbf{r} \\
\Delta b
\end{array}\right)
$$

The corresponding system of equations is usually overdetermined $(n>4)$, with design matrix A containing the satellite unit vectors $\mathbf{u}^{i}$ ( $i=1$ to $n$ ) and leastsquares solution (unit weight matrix)

$$
\Delta \mathbf{x}=\left(\mathbf{A}^{T} \mathbf{A}\right)^{-1} \mathbf{A}^{T} \mathbf{\Delta} \mathbf{P}
$$

We further developed our understanding of the influence of the ionosphere on positioning on a particular case of the problem (Mohino 2008). This particular case involves directly ionospheric delays $I^{i}$ in place of the observations $\Delta P^{i}$ producing positioning errors induced only by the ionosphere (Mohino 2008). It enables the development of the matrix $\left(\mathbf{A}^{T} \mathbf{A}\right)^{-1} \mathbf{A}^{T}$ to obtain analytical expressions binding ionospheric delays to positioning errors through a fictitious highly symmetrical satellite distribution. This distribution consists of one zenithal satellite and an even number of satellites at constant elevation $\eta$ uniformly distributed in azimuth. The simplest example of such a distribution comprises five satellites, one at the zenith and the others towards each cardinal direction (Fig. 10). 


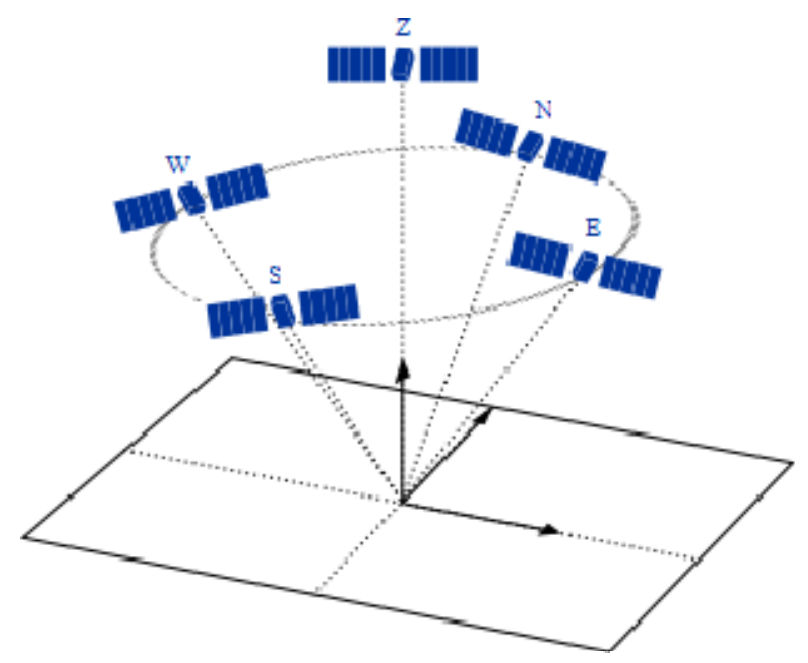

Fig. 10 Fictitious highly symmetrical distribution of 5 satellites. A satellite sky distribution involving 5 fundamental directions (northwards $-\mathrm{N}$, eastwards $-\mathrm{E}$, southwards $-\mathrm{S}$, westwards$\mathrm{W}$-and at the zenith-Z) yields the simplest analytical expressions binding ionospheric delays to positioning errors.

This particular case yields simple formulas for horizontal errors:

$$
\Delta N=\frac{1}{2 \cos \eta}\left(I^{S}-I^{N}\right)
$$

and

$$
\Delta E=\frac{1}{2 \cos \eta}\left(I^{W}-I^{E}\right)
$$

Indeed the north (resp. east) component depends only on the ionospheric delaysor corresponding TEC-along the north (resp. east) axis. Furthermore, the horizontal errors relate not only on TEC but on its gradients.

Based on this reasoning, hourly means of horizontal errors and TEC gradients supply a useful interpretation of the observed error distribution (Fig. 11). At mid-latitudes, these statistics disclose the effect of larger TEC values toward the equator with the largest gradients around local noon, leading to a north bias without proper correction. Following sun course, their profile along the east axis corresponds to a larger TEC eastwards in the morning and westwards in the afternoon. The combination of north and east errors explains the local-time profile of the uncorrected horizontal accuracy, a concave curve from north error with modulations in the morning and evening from east error (similar to Fig. 7). This 
profile is also depicted by Allain and Mitchell (2009) performing absolute positioning using precise products.

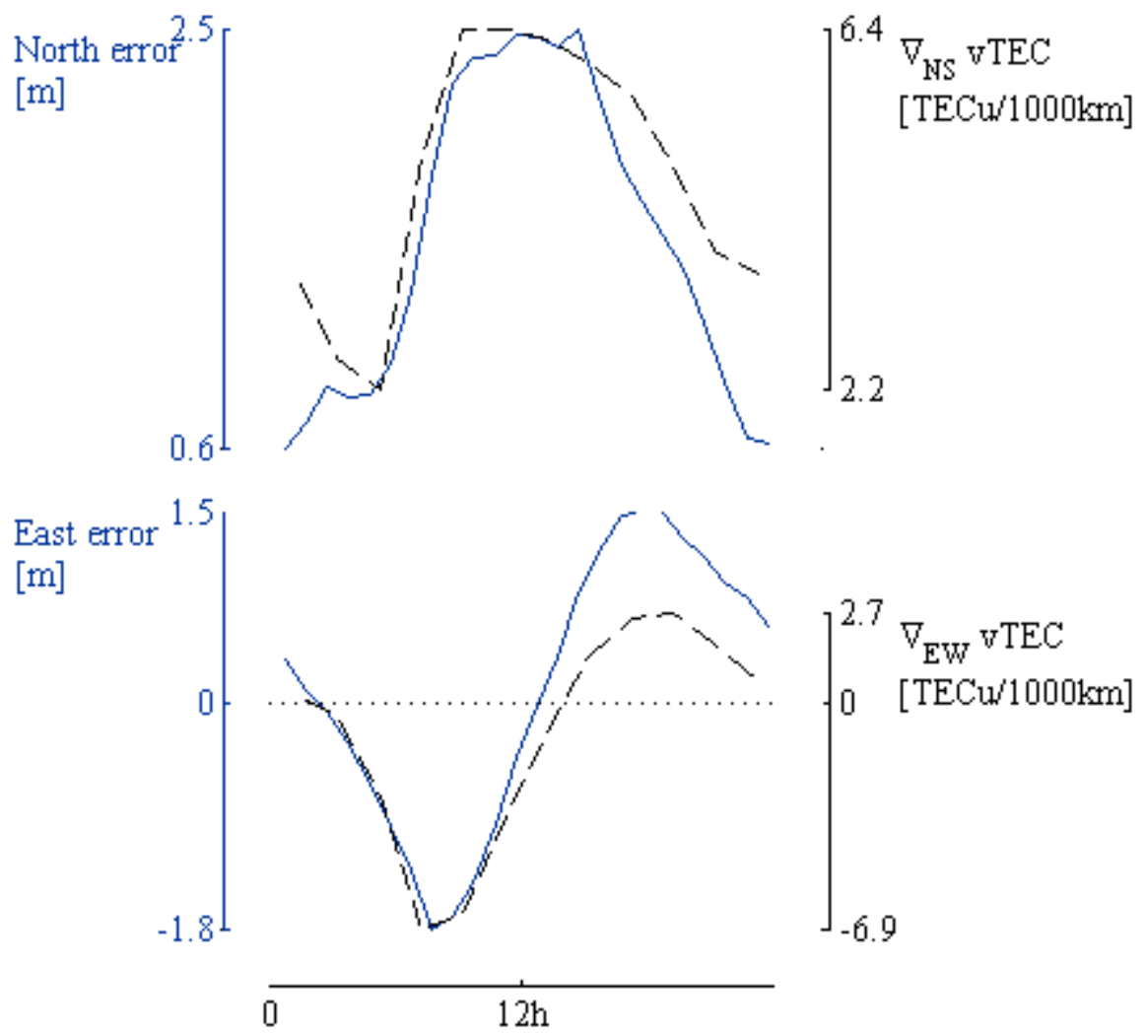

Fig. 11 Relations between the north (top plot) and east (bottom plot) uncorrected positioning errors (solid lines) and the north-south and east-west TEC gradients $\nabla T E C$ (dashed lines) at Brussels. These statistics result from the breakdown of the positioning errors dataset in 1-hour bins and the computation of TEC from 2-hour IGS GIM at ionospheric pierce points at about $666 \mathrm{~km}$ from the station toward each cardinal direction.

\section{Horizontal discrepancies left by the corrections}

As for the uncorrected horizontal accuracy, the mean north error constitutes an important driver of the corrected horizontal solutions. These solutions would indeed become significantly more accurate if the models eliminated the north bias. Unfortunately this bias persists and deteriorates the horizontal accuracy when it increases in absolute value (Fig. 12). It is indeed insufficiently corrected with the Klobuchar algorithm but overcorrected with NeQuick with its associated negative mean north errors. 


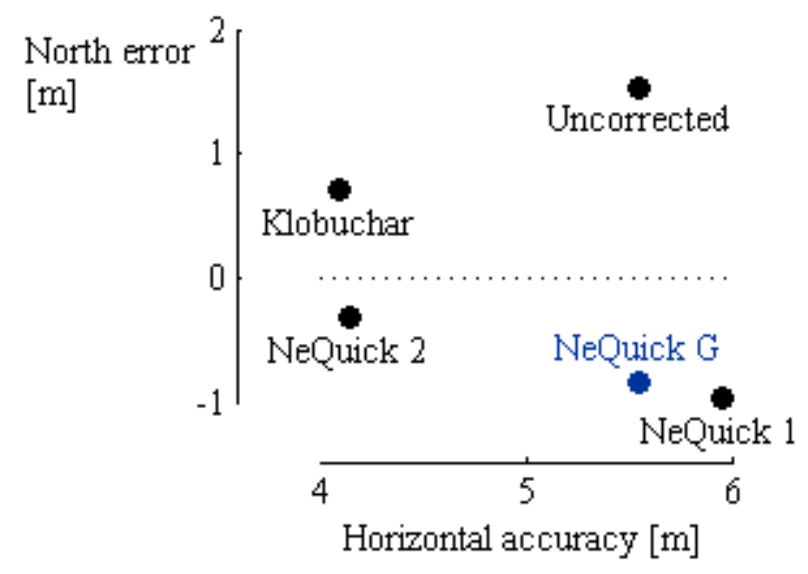

Fig. 12 Relation between the north error and the horizontal accuracy obtained with and without the ionospheric corrections at Brussels. Decreasing absolute values of the north error correspond to enhanced horizontal accuracies.

Similar conclusions prevail for the other stations included in the global performances description. The largest absolute values of the mean uncorrected north error (Fig. 13) coincide with the worst uncorrected horizontal accuracies (Fig. 8) at Cape Ferguson and Puerto Rico. Puerto Rico is also the only station where NeQuick $G$ produces worse results than NeQuick 1 both in horizontal accuracy and north error. The obtained positive (resp. negative) north bias in the northern (resp. southern) hemisphere matches the background southwards (resp. northwards) TEC gradients. It is not totally removed by the Klobuchar algorithm and, on the contrary, reversed by the NeQuick model except at Fortaleza. Noticeably NeQuick 1 amplifies the north error at this low-latitude station. There the horizontal positioning statistics for the other cases resemble more those of a north mid-latitude station. Fortaleza is indeed located under the north slope of the southern crest of the equatorial anomaly where TEC gradients point to the south. 


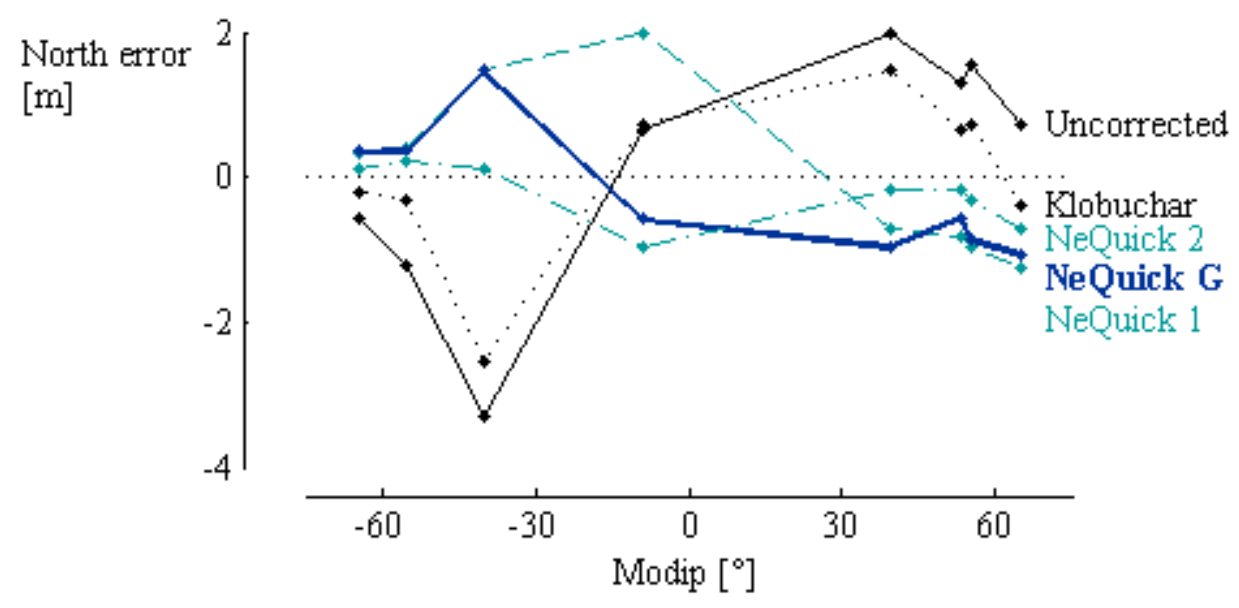

Fig. 13 Modip variability of the north error obtained with and without the ionospheric corrections. The NeQuick model (thick line for NeQuick G, dashed for NeQuick 1 and dash-dotted for NeQuick 2) overcorrects the north bias (continuous line) generally positive (resp. negative) in the north (resp. southern) hemisphere with the exception of Fortaleza. Inversely the Klobuchar algorithm (dotted line) undercorrects it.

Focusing on the horizontal discrepancies of the Galileo correction, the apparent north-south gradients mismodeling from NeQuick G should be further investigated for its key role in the limited horizontal positioning improvement despite the rather effective sTEC modeling. As an example, at Brussels for the worst month of the year 2002 (Fig. 6), the horizontal error distribution appears skewed southwards, which influences the most the 95th percentile (Fig. 14, left plot). Considering the original north bias, this effect highlights the mean overcorrection from NeQuick G along the north axis. It results from a mean sTEC overestimation toward south and the absence of satellites in a portion of the north sector (Fig. 14, right plot). 

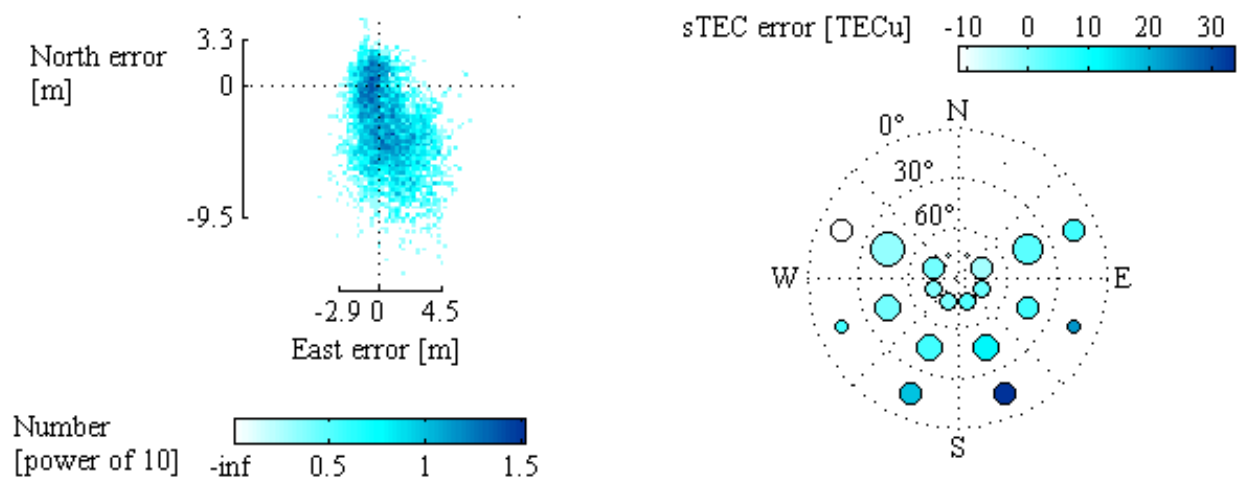

Fig. 14 Comparison between the distributions of horizontal errors (left plot) and sTEC residual errors (right plot) at Brussels in March 2002. NeQuick G induces a horizontal mean error southwards consistent with a sTEC mean overestimation in the same direction. The axes on the left plot represent 2- $\sigma$ intervals around the corresponding means. The sTEC errors on the right plot correspond to mean differences with measured values for $30^{\circ}$ elevation and $45^{\circ}$ azimuth bins and the circle surface to the number of values in each bin.

\section{Potential origins of Galileo correction discrepancies}

The Galileo single frequency ionospheric correction proceeds from an algorithm in three steps, each of them potentially impacting the correction performances. The first step takes place at each GSS. It consists in measuring the sTEC and adapting the NeQuick model to measurements via a modulation of the solar activity input. Accommodating the model intrinsic weaknesses, it provides a daily effective ionization level $A z$ for each GSS. The second step supplies the navigation message for the next day with 3 coefficients from the interpolation of all the $A z$ values against modip (1). The third step is performed by the receiver: The receiver runs the NeQuick model for each satellite in view together with the $A z$ value computed at its location from the broadcast coefficients.

This section distinguishes different effects from the combination of NeQuick intrinsic mismodeling and adapted data characteristics through the correction algorithm. It discusses the sensitivity to the effective ionization level in space and time conditioning the accessible optimization range at each GSS as well as the impact of $\mathrm{Az}$ interpolation errors. Then it examines the influence of the use of sTEC data for model adaptation. Finally, it addresses the distortion of the electron density profile consecutive to TEC adaptation. 


\section{Effective ionization level sensitivity}

The Galileo correction relies on the effectiveness of the data adaptation technique. In this case, this technique determines the effective ionization level that minimizes the RMS difference between a daily subset of sTEC measurements and their corresponding modeled value (Nava et al. 2006). It depends on the location and day considered.

Similar $A z$ variations induce different TEC modifications at different locations. For a given day, the variation from an initial value-for example, the daily solar flux at $10.7 \mathrm{~cm}$-providing the adequate TEC at one station possibly implies large biases at others (Fig. 15, top plot). At Brussels modip in 2002, feeding NeQuick $G$ with the daily solar flux leaves a yearly mean vertical TEC (vTEC) underestimation. This underestimation fades-yet not totally-thanks to sTEC adaptation at Brussels (Bidaine and Warnant 2011). However, daily adaptation of Brussels sTEC data results in effective ionization levels imposing a large overestimation at smaller modip in absolute value. Furthermore, it reaches minimum RMS differences involving a number of modeled STEC values on average overestimated southwards (from Brussels) and underestimated northwards. Hence, it produces excessive north-south TEC gradients. 


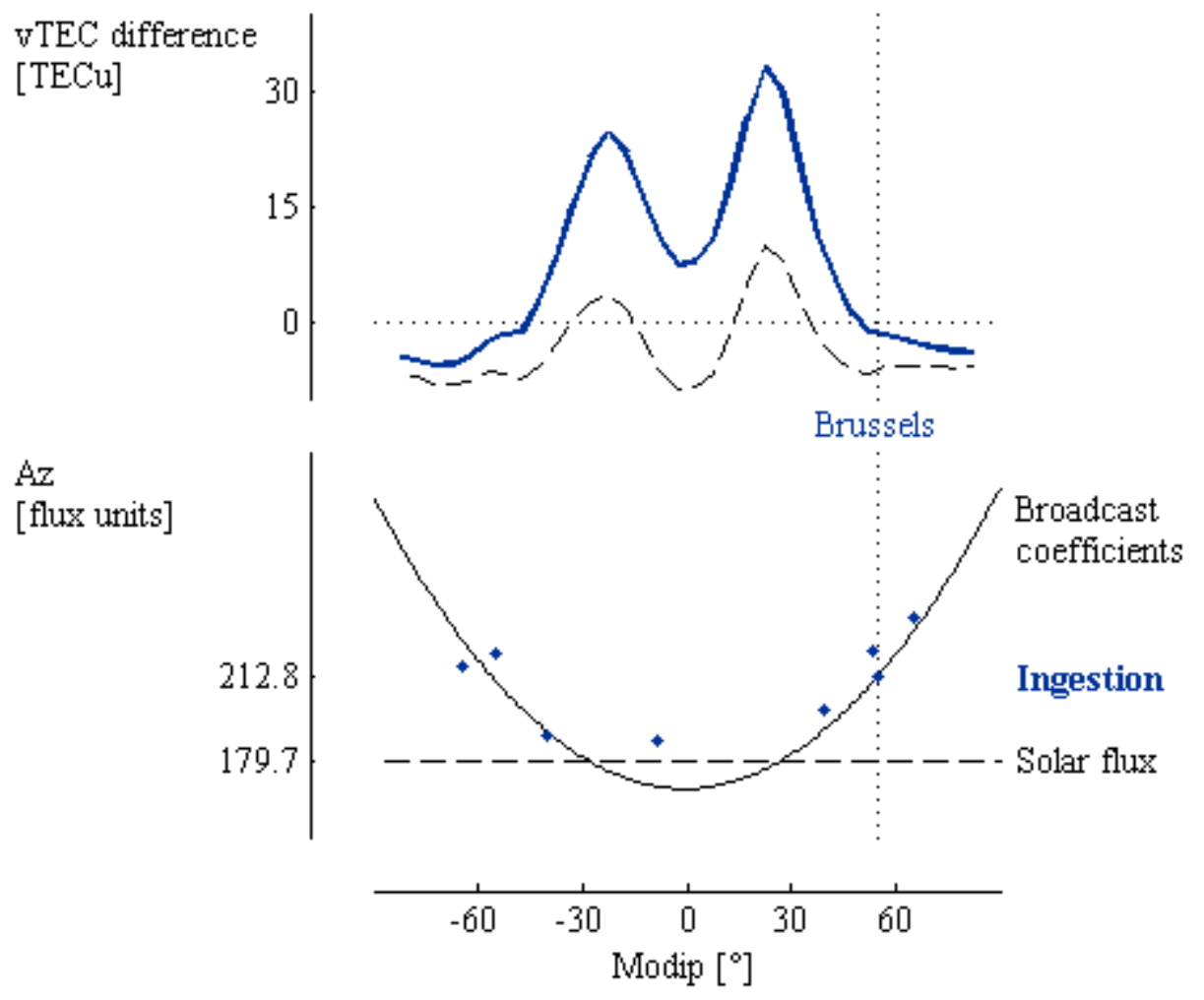

Fig. 15 Difference between IGS GIM and NeQuick G vTEC for different solar activity input parameters (top plot) and comparison between such parameters mainly obtained from sTEC adaptation (bottom plot). On the one hand, the yearly mean vTEC difference presents southwards gradients around Brussels (top plot) using the daily effective ionization level computed at Brussels from simulated broadcast coefficients or sTEC adaption (thick line). On the other hand, yearly means of daily $A z$ values (bottom plot) obtained from sTEC adaption at the test stations (dots) are well approximated by the parabola defined by the broadcast coefficients (continuous line). The yearly mean of the daily solar flux (dashed line in bottom plot) considered as an initial value for data adaptation generally yields a vTEC underestimation apart from low latitudes (dashed line in top plot).

Introduced to fit the observed $A z$ variability in the geomagnetic northsouth direction, the parabolic interpolation adjusting the broadcast coefficients renders fairly good sTEC performances in the RMS sense (Fig. 2) but a biased representation of TEC gradients affecting horizontal positioning. At Brussels in 2002, it corresponds on average to the value obtained from sTEC adaptation (Fig. 15, bottom plot) and delivers similar TEC results. Hence, both $A z$, larger than for lower latitudes, produce excessive sTEC values on average toward the equator (Fig. 14). At Fortaleza, the mean interpolated effective ionization level differs significantly from the optimal value leading to underestimated vTEC. Yet it 
allows smaller TEC gradients errors and a better horizontal accuracy. The largest horizontal discrepancies occur at low mid-latitudes (Fig. 8) where average gradients along the north axis are the most stretched.

At last, the 1-day latency in the dissemination of the broadcast coefficients — computed from sTEC measurements of the day before-has a minor effect on positioning performances, unlike TEC (Bidaine and Warnant 2011). The yearly sTEC RMS error at Brussels in 2002 increases by $20 \%$ when using NeQuick G with the interpolated effective ionization level instead of the current day $A z$ value. On the contrary, the horizontal accuracy remains unchanged. Nevertheless, the intra-day variability of TEC horizontal gradients (Fig. 11) supposes also a variable effectiveness of the TEC adaption at different hours of local time.

\section{Data characteristics}

Besides the obvious influence of the elevation on sTEC adaptation, the calibration method applied to dual-frequency phase geometry-free combinations deserves more concern. This method determines the unknown numbers of cycles involved in phase measurements called ambiguities. It computes the average differences on a continuous set of epochs between these measurements and sTEC estimates from another source. In this study, it relies on GIM to collect reference sTEC (Orus et al. 2007b). More often, it uses code measurements, noisier and affected by satellite and receiver Differential Code Biases (DCB), and is then referred to as carrier-to-code leveling. According to Conte et al. (2011), this technique may cause errors of the order of 8 TECU on estimated DCB at high solar activity. Implemented following Warnant and Pottiaux (2000), it supplies sTEC data 6.8 TECU smaller on average than the first technique at Brussels in 2002 (Bidaine and Warnant 2009). Moreover, these data used for NeQuick G adaptation at that station in that year yield a smaller modeled sTEC, less stretched horizontal TEC gradients and better positioning performances (Fig. 16). Hence, these performances would be further improved for more accurate TEC monitoring procedures potentially ensuing from triple-frequency combinations (Spits and Warnant 2011). 


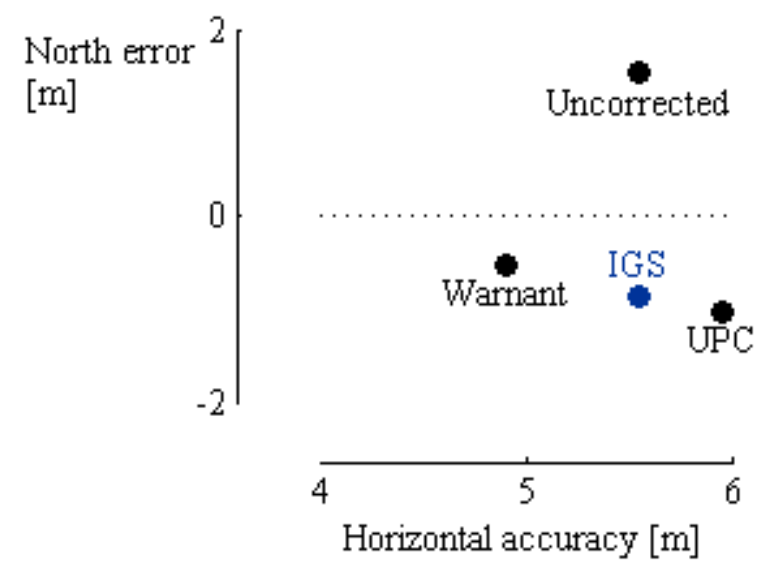

Fig. 16 Relation between the north error and the horizontal accuracy obtained with NeQuick G at Brussels from the adaptation to different sTEC data sets. The horizontal accuracy improves and the absolute mean north error decreases when using sTEC data from carrier-to-code leveling (Warnant and Pottiaux 2000) instead of GIM calibration (Orus et al. 2007b).

\section{Electron density profile distortion}

The main advantage of the NeQuick model regarding TEC modeling lies in its 3D representation of the ionosphere. Avoiding mapping functions and associated drawbacks, this representation-often called "profiling"-renders TEC in a specific direction from the integration through vertical electron density profiles. These profiles consist in analytical functions of which the characteristics are obtained from peak parameter values. In this respect, the main peak of the profile (in the F2 layer) constitutes a strong driver of the model performances.

sTEC adaptation provokes a distortion of the electron density profile. NeQuick G profile utilizes a too simplistic suboptimal formulation for the topside, the upper part of the ionosphere, like NeQuick 1 (Bidaine and Warnant 2010). Compensating for this intrinsic mismodeling when adapting TEC, the profile is stretched in height and electron density following its uplifted over-ionized F2 peak (Fig. 17). Obtained from sTEC adaptation at Dourbes, located $80 \mathrm{~km}$ south from Brussels, in 2002 and compared to Dourbes digisonde measurements, it adopts first too weak then too strong electron densities for increasing fixed heights. 


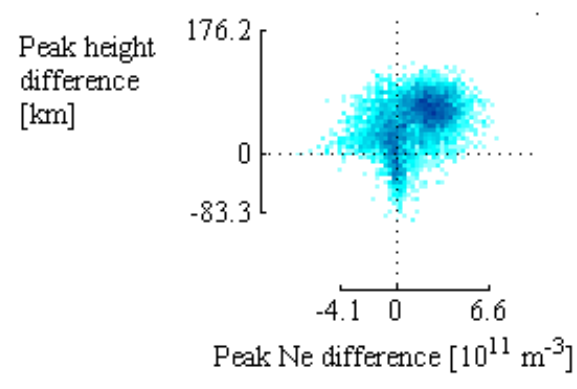

Number

[power of 10]

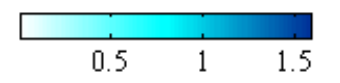

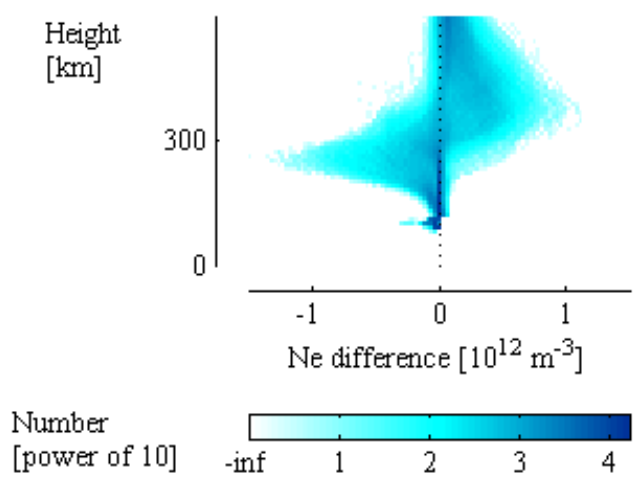

Fig. 17 Distributions of the differences of peak electron density and height (left plot) and electron density profile (right plot) between hourly manually scaled data from Dourbes digisonde and NeQuick G. sTEC data adaptation generally occasions an uplifted over-ionized peak of the electron density profile (left plot). This profile is consequently stretched with underestimated electron densities at fixed lower heights and overestimated concentrations at larger altitudes (right plot). The limited portion of the topside actually not probed but modeled by the digisonde is only shown for illustration.

The model intrinsic modifications underpin the observed favorable evolution from one version of NeQuick to the next. By comparison to NeQuick 1, NeQuick G benefits from simplifications in the bottomside (Leitinger et al. 2005) and updated modip information. In particular, it improves low latitude performances, for example, at Fortaleza (Figs. 8, 13). However, it remains affected by the aforementioned topside limitation also identified by Stankov et al. (2007). This limitation relates to a twofold formula describing a coefficient shaping the topside height-dependent thickness parameter. It generates different effects during the two corresponding periods of the year, from April to September and from October to March, which become more homogeneous with NeQuick 2 (Fig. 6). This latest version has indeed received a new topside shape parameter formula based on topside soundings (Coisson et al. 2006).

\section{Conclusion and perspectives}

Simulating the Galileo single frequency ionospheric correction, we analyzed its performances at different stations in 2002. In addition to the official Galileo version of the underlying NeQuick model, we built hypothetical counterparts on two other versions and we compared their results to the Klobuchar algorithm implemented in the GPS. 
We obtained 32, 31 and 28 \% sTEC RMS residual errors for successive NeQuick versions, 50 \% for the Klobuchar algorithm and a 92-94 \% compliance of NeQuick with the Galileo algorithm specifications (residual error smaller than $30 \%$ of the actual sTEC or 20 TECU, whichever is larger). Looking to positioning errors, we observed a significant vertical accuracy improvement from the ionospheric corrections (95th percentiles between 9 and $11 \mathrm{~m}$ vs. $25 \mathrm{~m}$ without correction) but comparable horizontal accuracies with and without correction (between 5 and $7 \mathrm{~m}$ residual errors with a maximum for the uncorrected case). As reaffirmed through our spatio-temporal variability study, positioning accuracies, although influenced by similar drivers, do not simply follow sTEC performances.

Focusing on Brussels, we put these observations into perspective forming our intuition on a particular case of the positioning mathematical problem. Considering a fictitious highly symmetrical satellite distribution, we depicted the effect of TEC gradients on horizontal positioning errors, emphasizing the role of the north component. In this respect, systematic mismodeling materializing the difference between accuracy and precision should not be neglected.

Finally concentrating on Galileo correction horizontal discrepancies, we discussed how sTEC data adaptation transposes the limitations of the modeled electron density profile-mainly related to the topside representation - in other products. We associated the effective ionization level spatial sensitivity to the detected excessive north-south TEC gradients, and we addressed the sTEC measurement technique. Obviously, optimizing against one criterion may yield unpredictable effects on unconsidered features.

Benefiting from this background possibly reviewed during the In-Orbit Validation phase of Galileo, we will further address single frequency users' needs for an effective ionospheric correction. We will envisage possible alternatives to the current definition of the Galileo algorithm, among which regional procedures. 
Acknowledgments The work presented is part of $\mathrm{B}$. Bidaine's $\mathrm{PhD}$ thesis in progress under a F.R.S.-FNRS fellowship (Belgian National Fund for Scientific Research). We would like to acknowledge S. Radicella and B. Nava from ICTP in Trieste for providing us NeQuick latest version; R. Prieto-Cerdeira and R. Orus from ESA/ESTEC for the TEC data as well as the official NeQuick version for Galileo.

\section{References}

Allain D, Mitchell C (2009) Ionospheric delay corrections for single-frequency GPS receivers over Europe using tomographic mapping. GPS Solut 13 (2):141-151. doi:10.1007/s10291-0080107-y

Bidaine B, Warnant R Measuring Total Electron Content with GNSS: Investigation of Two Different Techniques. In: 11th International Conference on Ionospheric Radio Systems and Techniques (IRST 2009), Edinburgh (UK), April 28th to May 1st, 20092009. Institution of Engineering and Technology-IET, London (UK), pp 201-206. doi:10.1049/cp.2009.0063

Bidaine B, Warnant R (2010) Assessment of the NeQuick model at mid-latitudes using GNSS TEC and ionosonde data. Adv Space Res 45 (9):1122-1128. doi: $10.1016 /$ j.asr.2009.10.010

Bidaine B, Warnant R (2011) Ionosphere modelling for Galileo single frequency users: illustration of the combination of the NeQuick model and GNSS data ingestion. Adv Space Res 47 (2):312-322. doi:10.1016/j.asr.2010.09.001

Bruyninx C (2004) The EUREF Permanent Network: a multi-disciplinary network serving surveyors as well as scientists. GeoInformatics 7:32-35

Coisson P, Radicella SM, Leitinger R, Nava B (2006) Topside electron density in IRI and NeQuick: Features and limitations. Adv Space Res 37 (5):937-942. doi:10.1016/j.asr.2005.09.015

Conte J, Azpilicueta F, Brunini C (2011) Accuracy assessment of the GPS-TEC calibration constants by means of a simulation technique. J Geodesy:1-8. doi:10.1007/s00190-011$\underline{0477-8}$

Dow JM, Neilan RE, Rizos C (2009) The International GNSS Service in a changing landscape of Global Navigation Satellite Systems. J Geodesy 83 (3-4):191-198. doi:10.1007/s00190008-0300-3

Hernández-Pajares M, Juan JM, Sanz J, Orus R, Garcia-Rigo A, Feltens J, Komjathy A, Schaer SC, Krankowski A (2009) The IGS VTEC maps: a reliable source of ionospheric information since 1998. J Geodesy 83 (3-4):263-275. doi:10.1007/s00190-008-0266-1

Hernandez-Pajares M, Juan JM, Sanz J, Ramos-Bosch P, Rovira-Garcia A, Salazar D, VenturaTraveset J, Lopez-Echazarreta C, Hein G The ESA/UPC GNSS-Lab tool (gLAB): an advanced multipurpose package to process and analyse GNSS data. In: 5th ESA Workshop on Satellite Navigation User, Noordwijk (The Netherlands), December 8th to 10th, 20102010.

Hofmann-Wellenhof B, Lichtenegger H, Wasle E (2008) GNSS - Global Navigation Satellite Systems - GPS, GLONASS, Galileo, and more. Springer, Vienna. doi:10.1007/978-3211-73017-1

Klobuchar JA (1987) Ionospheric Time-Delay Algorithm for Single-Frequency GPS Users. IEEE T Aero Elec Sys AES-23 (3):325-331. doi:10.1109/TAES.1987.310829

Leitinger R, Zhang ML, Radicella SM (2005) An improved bottomside for the ionospheric electron density, model NeQuick. Ann Geophys-Italy 48 (3):525-534

Mohino E (2008) Understanding the role of the ionospheric delay in single-point single-epoch GPS coordinates. J Geodesy 82 (1):31-45. doi:10.1007/s00190-007-0155-z

Nava B, Coisson P, Radicella SM (2008) A new version of the NeQuick ionosphere electron density model. J Atmos Sol-Terr Phys 70 (15):1856-1862. doi:10.1016/j.jastp.2008.01.015

Nava B, Radicella SM, Leitinger R, Coisson P (2006) A near-real-time model-assisted ionosphere electron density retrieval method. Radio Sci 41 (6):RS6S16. doi:10.1029/2005rs003386 
Orus R, Arbesser-Rastburg B, Prieto-Cerdeira R, Hernandez-Pajares M, Juan JM, Sanz J (2007a) Performance of Different Ionospheric Models for Single Frequency Navigation Receivers. Paper presented at the 2007 Beacon Satellite Symposium, Boston (USA),

Orus R, Cander LR, Hernandez-Pajares M (2007b) Testing regional vertical total electron content maps over Europe during the 17-21 January 2005 sudden space weather event. Radio Sci 42 (3):RS3004. doi:10.1029/2006rs003515

Prieto-Cerdeira R, Orus R, Arbesser-Rastburg B Assessment of the Ionospheric correction algorithm for GALILEO Single Frequency Receivers. In: 3rd ESA Workshop on Satellite Navigation User Equipment Technologies NAVITEC 2006, Noordwijk (The Netherlands), December 11th to 13th, 20062006.

Radicella SM, Leitinger R (2001) The evolution of the DGR approach to model electron density profiles. Adv Space Res 27 (1):35-40. doi:10.1016/S0273-1177(00)00138-1

Rawer K (1963) Propagation of Decameter Waves (HF-Band). In: Landmark B (ed) Meteorological and Astronomical Influences on Radio Wave Propagation, vol 11. Academic Press, New York (USA), pp 221-250

Snay RA, Soler T (2008) Continuously Operating Reference Station (CORS): History, Applications, and Future Enhancements. J Surv Eng-ASCE 134 (4):95-104. doi:10.1061/(Asce)0733-9453(2008)134:4(95)

Spits J, Warnant R (2011) Total electron content monitoring using triple frequency GNSS: Results with Giove-A/-B data. Adv Space Res 47 (2):296-303. doi:10.1016/j.asr.2010.08.027

Stankov SM, Marinov P, Kutiev I (2007) Comparison of NeQuick, PIM, and TSM model results for the topside ionospheric plasma scale and transition heights. Adv Space Res 39 (5):767-773. doi:10.1016/j.asr.2006.10.023

Warnant R, Pottiaux E (2000) The increase of the ionospheric activity as measured by GPS. Earth Planets Space 52 (11):1055-1060

\section{Author Biographies}

Benoît Bidaine, a F.R.S.-FNRS Research Fellow, currently finishes his $\mathrm{PhD}$ about ionosphere modeling for Galileo single frequency users at the University of Liège (Belgium). He undertook this research within the Geomatics Unit after an internship at the European Space Agency (ESA) in 2006, at the end of his physics engineering master awarded by the University of Liège.

Matthieu Lonchay also received a F.R.S.-FNRS Research Fellowship to investigate ionospheric effects on GNSS, on Precise Point Positioning in particular. Holding an MSc in geographical sciences since 2009, he is currently visiting the University of Nottingham thanks to the ODISSEA Prize he has been awarded by the Belgian Senate for his master thesis.

Prof. René Warnant has recently been appointed full-time Professor at the University of Liège in the field of geodesy and GNSS. He obtained a PhD from the Catholic University of Louvain in 1996, worked at the Royal Observatory of Belgium and the Royal Meteorological Institute of Belgium, has been involved in several COST actions and is a member of ESA GNSS Science Advisory Committee (GSAC). 